\title{
Multiple Vaccines: How Do We Choose?
}

\author{
Neil B. Minkoff, MD
}

\begin{abstract}
BACKGROUND: As preventive medicine is a cornerstone of managed care, most health plans have traditionally featured automatic vaccine coverage routed through the medical benefit. However, with the advent of emerging vaccines, managed care stakeholders must revise decision-making processes and choose among multiple products targeting the same disease.
\end{abstract}

OBJECTIVE: To review the motivating forces behind traditional vaccine coverage in managed care and discuss the need for managed care organizations (MCOs) to subject their vaccine policies to greater examination in the changing landscape of emerging vaccines.

SUMMARY: While variable vaccine coverage or choices in vaccine coverage is a relatively novel concept in managed care, the evaluation of vaccines in this setting is usually most effectively performed via a traditional route for MCOs: the Pharmacy \& Therapeutics (P\&T) committee. In some cases, a technology assessment committee is a more appropriate avenue for evaluation, depending on the disease state, administration, and plan infrastructure. Through these routes of evaluation, criteria similar to those used for other pharmaceutical agents under review should be employed in the review of vaccine options. The primary criteria evaluated include safety, efficacy, cost, and value. In addition, a set of miscellaneous factors must also be considered, including both tangible and intangible components. For example, the relevance of an agent to the specific covered population, compliance costs offsets, quality-of-life considerations, and both patient and provider demand should all be taken into account. Human papillomavirus vaccination provides a pragmatic example for applying the aforementioned strategy for vaccine evaluation in managed care.

CONCLUSION: The changing landscape of vaccine coverage in managed care, particularly in the availability of novel agents, demonstrates a need for MCOs to subject their vaccine policies to much greater examination. Through traditional avenues such as P\&T and technology assessment committees, stakeholders should seek to evaluate standard criteria such as safety, efficacy, and cost-effectiveness, with additional considerations made for factors unique to the preventive nature of vaccines.

KEYWORDS: Vaccine, P\&T, HPV, Safety, Effectiveness, Cost

J Manag Care Pharm. 2007;13(7)(suppl S-b):S16-S20

Copyright@ 2007, Academy of Managed Care Pharmacy. All rights reserved.

\section{Author}

NEIL B. MINKOFF, MD, is medical director, and pharmacy, Harvard Pilgrim Health Care, Wellesley, Massachusetts.

AUTHOR CORRESPONDENCE: Neil B. Minkoff, MD, Medical Director, Network Services and Pharmacy, Harvard Pilgrim Health Care, 93 Worcester St., Wellesley, MA 02481. Tel: (617) 509-0170; Fax: (617)

509-9144; E-mail:neil_minkoff@harvardpilgrim.org
I $\mathrm{n}$ choosing between multiple vaccine options, managed care stakeholders currently have few definitive answers. This is due, in part, to the fact that variable vaccine coverage or choice in vaccine coverage is a relatively new concept in the industry. As preventive medicine is a cornerstone of managed care, most plans have traditionally featured automatic vaccine coverage routed through the medical benefit. ${ }^{1}$ In fact, just 8 years ago, the National Vaccine Advisory Committee (NVAC) sponsored an article published in the Journal of the American Medical Association, pointing out the near-universal coverage of vaccines by managed care organizations (MCOs). ${ }^{1,2}$ Members traditionally had little or no cost share, depending on whether or not there was an office visit with the administration, and plans rarely chose preferred products or implemented any contracting or rebating. Instead, decisions were based on current Advisory Committee on Immunization Practices (ACIP) recommendations. If ACIP recommendations were in any way unclear or required augmentation, plans would seek the advice of different specialty groups such as the American Academy of Pediatrics.

These supplemental recommendations tended to be brought to MCO technology assessment committees (i.e., the medical benefit version of pharmacy and therapeutics committees) and were, for the most part, implemented as the benefits offered by the plan. This focus on promoting preventive medicine complemented the birth of managed care in general, particularly since health maintenance organization (HMO) models with fully insured membership was the norm during this formative period. This developmental period in managed care also featured the introduction of population management-looking beyond just the interaction between the physician and the member to a broader mix of patient types-and the early concepts of longitudinal care. Vaccines were a very obvious place to begin this level of preventive care, and the administration of these agents fit very well with the philosophies and the practices upon which managed care and HMOs were being built.

In addition to the preventive benefit of these agents, the historically universal coverage of vaccines in managed care can be attributed to the extra layer of complexity added when attempting to manage these agents due to state mandates. Although these mandates feature a significant amount of overlap (e.g., requirements for the coverage of pediatric vaccines), differences exist from state to state in terms of vaccine requirements and what each state provides. For example, in Massachusetts most of the vaccines administered by pediatricians are supplied to the doctor cost-free by the state and administered as part of the public health mission of the state. ${ }^{3}$ This added layer of complexity requires that Massachusetts MCOs know which vaccines are the responsibility of the MCO and which are provided thorough public funding. The gap between what the 
state mandates and what the state will fund has been a significant concern to MCOs. However, many MCOs have settled on the position of covering this gap and end up having variable coverage rules in each state in which they participate.

\section{Shifting Paradigm}

The traditional notion of universal vaccine coverage in managed care is changing. In turn, managed care stakeholders must be aware of the reasons for this trend and devise strategies to deal with the shifting paradigm.

Much of the need for vaccine decision making in managed care is the result of novel vaccines that have been recently released or vaccines currently in the pipeline. The need for vaccine management in managed care will only increase as time goes on, with advances in drug development predicting hundreds of disease states, including chronic infections such as human immunodeficiency virus and noninfectious conditions such as Alzheimers, whose illness burden could be potentially lessened by vaccination. In addition, more combination products are being developed and becoming available.

This is of particular interest from a formulary management perspective, since pharmacy and therapeutics (P\&T) committee members will have to ascertain if specific combination vaccines are more efficacious than their respective individual counterparts and ultimately worth the additional cost. Furthermore, advancements in vaccine technology have provided potential benefits in terms of effectiveness, but this comes at a higher cost; $\mathrm{P} \& \mathrm{~T}$ committee members will again need to determine if the additional clinical benefit justifies the increased cost in these cases. These cost considerations also come into play with newer, recently introduced single vaccines, which carry a generally high cost, in addition to combination products. This becomes even more significant when plans compare the cost of recently introduced vaccines with traditional ones that are generally less costly and sometimes provided by the state. At Harvard Pilgrim in Wellesley, MA, for example, coverage of the quadrivalent human papillomavirus (HPV) vaccine (Gardasil) will result in an increase in medical spending, which may in turn lead to rising premiums and future problems in terms of coverage issues and what employers choose to fund.

In addition to the clinical and financial components being evaluated in the coverage of recently introduced vaccines is the question of scope from an individual and population perspective. While coverage of traditional vaccines was often a matter of public health (e.g., influenza), many recently introduced and pipeline vaccines target conditions that are mainly relevantonanindividuallevel(e.g., herpeszoster). Whiletraditional vaccines have led to the virtual eradication of highly contagious communicable diseases, including those that involve mass mortality, some of the newer vaccines are much more focused on personal health. As a result, the public health component is beginning to be removed from vaccine management as managed care increasingly moves toward the individual state.

The introduction of new vaccines has also initiated the issue of competition between agents targeting the same disease state. The live intranasal influenza vaccine (FluMist) provides an excellent example of an agent with competitors that has been given significant consideration. Initially, vaccination with the live intranasal influenza vaccine was not embraced by many organizations because of a noncompetitive price and concerns over the use of a live rather than attenuated virus. While MCOs wanted to encourage as many members as possible to get immunized against influenza for herd immunity, concerns remained regarding members who most needed to be immunized (i.e., elderly patients, pediatric patients, and immuno-suppressed patients) not receiving a live virus.

Data from a 2006 clinical study demonstrating safety and immunogenicity for the live intranasal influenza vaccine in the pediatric population of patients down to the age of 1 year provides additional support for the live intranasal vaccine, which is now being factored into coverage considerations. ${ }^{4}$ In addition, the cost of the live intranasal influenza vaccine has decreased, warranting further use of the agent in the organization. These recent developments have influenced MCOs to trust their clinicians to know which population should not have a live virus and to reserve the live virus for the members of the population that are needed to boost the herd immunity. In another disease state, these competitive considerations will come into play once more if GlaxoSmithKline's bivalent HPV vaccine (Cervarix) is approved as anticipated, since there is a quadrivalent HPV vaccine currently on the market.

\section{Managed Care Adaptation Strategies}

While variable vaccine coverage or choice in vaccine coverage is a relatively novel concept in managed care, the evaluation of vaccines in this setting is usually most effectively performed via a traditional route for MCOs: the P\&T committee. In some cases a technology assessment committee is a more appropriate avenue for evaluation, depending on the disease state, administration, and plan infrastructure. A hybrid of both P\&T and technology assessment committee evaluation is also an option, based on situational factors. Taking these routes of evaluation into consideration, adult vaccines should generally receive more scrutiny in terms of coverage than pediatric vaccines, mostly due to the fact that the more recently introduced adult vaccines have less of a public health orientation than pediatric vaccines. Furthermore, pediatricians have been a very vocal group in terms of quadrivalent $\mathrm{HPV}$ vaccine coverage since the product's approval, requiring many plans to change some of the manners in which they interact with their pediatric communities. The pediatric community is sensitive to coverage issues regarding vaccines since vaccines (a) generally do not face many of the cash flow issues that can occur with buying and billing by physicians and (b) have traditionally been available free from 
the state. At Harvard Pilgrim, issues surrounding the coverage of the quadrivalent HPV vaccine have affected the organization's relationship with its members and its network in a number of different ways. Regardless, the industry appears to have reached a consensus to start evaluating novel vaccines in the same manner as other pharmaceutical products that have come to market.

In evaluating the various vaccine options available to MCOs, criteria similar to those used for other pharmaceutical agents under review should be employed. The primary criteria evaluated include safety, efficacy, cost, and value. In addition, a set of miscellaneous factors must also be considered, including both tangible and intangible components. For example, the relevance of an agent to the specific covered population, compliance costs offsets, quality of life considerations, and both patient and provider demand should all be taken into account. These factors have significant managed care implications in the evaluation of recently introduced and pipeline vaccines. For example, if an organization starts making a push towards combination vaccines, reduced adolescent drop-off rates may result due to a fewer number of injections necessary. Combination vaccines may also positively affect quality of life for the same reasons. Considering it is much easier and much less expensive to keep a customer than to make a customer, patient demand should be taken into account in vaccine coverage evaluation.

Although the traditional criteria of safety, effectiveness, and cost must be factored into the evaluation of recently introduced vaccines, the depth and breadth of data used in the review of other pharmacotherapeutic agents is typically not available. A comprehensive level of reporting, such as that found in AMCP dossiers, is usually not possible for novel vaccines. When evaluating data on the safety of new vaccines entering the market, MCOs must often rely only on data submitted for U.S. Food and drug Administration approval and experience in other countries. With limited follow-up data available, the long-term effects of a recently approved vaccine are unknown. Similarly, MCOs must often rely only on submission data and experience in other countries in terms of effectiveness. While limited follow-up data evaluating effectiveness is a concern to both the private and public sectors, it becomes more of a significant issue to MCOs considering the long-range financial implications of a vaccine whose effectiveness declines as time goes on. Demonstrating the significance of declining effectiveness in the long-term, the live varicella vaccine (Varivax) was initially administered as a 1-time injection for the prevention of chicken pox. However, after postmarket experience demonstrated that protection against varicella declined over time, it became apparent that booster injections would be necessary. This was a clinically significant development to MCOs: children who initially had immunity against varicella through vaccination rather than virus exposure now required booster injections or risk developing more severe disease at an older age. ${ }^{5}$
Evaluation of the economic factors associated with vaccines is a new area for managed care. Whereas at one time vaccines were covered in a generally universal manner, stakeholders are now presented with a complex array of financial considerations. With a discounted average wholesale price (AWP) paid through the medical benefit and currently no member cost share or rebating, the upfront costs of vaccines are clear. However, considering that the value of vaccines lies in the prevention of disease and reduced expenses in the long term, cost offsets are not realized initially. Other factors must be taken into account as well, including the rise of unfunded mandates by states, which will result in increased costs to MCOs, and demand for recently introduced vaccines from patients, providers, and administrative service organization employers.

\section{An Example: HPV Vaccination}

HPV vaccination provides a pragmatic example for applying the aforementioned strategy for vaccine evaluation in managed care. As mentioned earlier, the market currently features 1 available quadrivalent vaccine for HPV prevention, but approval of a bivalent vaccine is anticipated in the near future. Considering the clinical and economic implications of cervical cancer in managed care, as well as the relatively high cost of the vaccines, this example highlights many of the difficulties MCOs will face in evaluating and managing emerging vaccines.

HPV remains a significant concern in managed care, with $>100$ types in total and $>30$ types being transmitted through sexual contact. ${ }^{6}$ Six million new genital HPV infections occur annually in the United States, but more importantly, persistent HPV infection is the leading cause of cervical dysplasia and neoplasia. ${ }^{6}$ In fact, $70 \%$ of cervical cancer is linked to types 16 and 18, and an estimated 10,000 women are diagnosed with the cancer every year. ${ }^{6}$ Considering the prevalence of HPV, the disease's association with cervical cancer, and uncertainty surrounding the protection offered by condoms against HPV, vaccination for HPV presents a significant opportunity for MCOs to potentially decrease mortality and avert costs.

In evaluating the vaccines targeting HPV for administration in a managed care population, stakeholders must first compare the clinical characteristics of each agent. To start, both the quadrivalent and bivalent HPV vaccines provide immunity against HPV types 16 and 18, the types responsible for $70 \%$ of cervical cancer. While the quadrivalent HPV vaccine also offers immunity against types 6 and 11 and thereby provides more genital wart protection, the ASO4 adjuvant employed by the bivalent HPV vaccine has demonstrated improved immune response and potentially improved cancer protection. ${ }^{7,8}$ Both vaccines have demonstrated excellent safety profiles and effectiveness for patients aged $>4$ years. Although no direct comparative data exist for the 2 products, researchers are currently recruiting patients for a head-to-head trial. ${ }^{9}$ Also, as pricing for the bivalent HPV vaccine has not yet been 
released, this component of the evaluation cannot yet be directly addressed.

Using only currently available public information, it is likely that both agents would be viewed favorably by a P\&T committee in terms of their safety profiles. The effectiveness discussion would center around the potential benefit of the bivalent HPV vaccine adjuvant versus the quadrivalent HPV vaccine adjuvant for types 6 and 11. With these 2 criteria at a virtual stalemate due to a lack of definitive data, cost-effectiveness would likely become the deciding factor.

In the evaluation of HPV vaccines, managed care stakeholders have to take into consideration several confounding variables that may have an impact on product utility or overall costs (see Figure). Among these are state mandates on vaccination requirements, which may dictate certain subpopulations that must be vaccinated, as well as the controversy related to mandatory vaccination, which may put customers at odds with their MCO. Also confounding the evaluation of HPV vaccines are questions regarding the long-term efficacy of the products, since necessary booster shots will increase costs. Concerns from groups who believe that HPV vaccination promotes promiscuity are being voiced, ${ }^{10}$ and this confounding variable will likely only increase as an additional product is approved and mandatory vaccination policies are put into place. Finally, administrative service organization employers present a unique situation where the MCO and the employer may have opposing views on patient benefit needs in terms of vaccination.

\section{Opportunities for MCOs}

Although managed care stakeholders face uncharted territory considering the rapidly evolving vaccine environment, opportunities similar to those employed in the management of other pharmacotherapeutic agents exist to lessen the impending economic burden. For example, contracts and rebating can be combined with a preferred product to reduce direct costs and promote the use of a specific vaccine. In terms of benefit design, a rider for the coverage of certain vaccines may be layered onto the basic insurance platform in specific situations without totally revamping current policies. The tiering of vaccines on formulary with variable member cost share may be used to promote the use of the most cost-effective product while still retaining patient choice. Members may also bear greater cost share for vaccines with less public health focus, and/or nonpreferred products, so as to encourage herd immunity and reduce costs.

\section{Conclusion}

The changing landscape of vaccine coverage in managed care, particularly in the availability of novel agents, demonstrates a need for MCOs to subject their vaccine policies to much greater examination. Through traditional avenues such as P\&T and technology assessment committees, stakeholders should seek to evaluate standard criteria such as safety, efficacy, and cost-effec-

\section{FIGURE Confounding Variables Associated With HPV Vaccination in Managed Care}

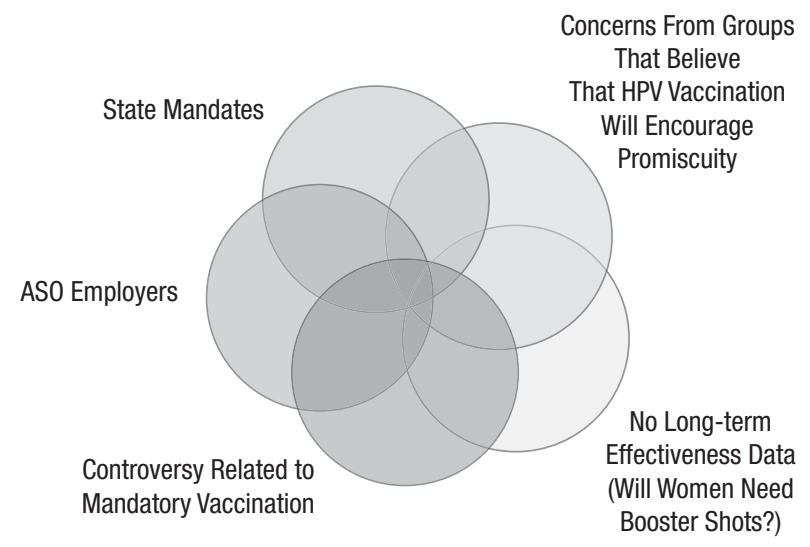

$A S O=$ administrative service organization; $H P V=$ human papillomavirus .

tiveness, with additional considerations made for factors unique to the preventive nature of vaccines. A greater deal of scrutiny should be placed on coverage for adult rather than pediatric vaccines since these agents are typically more individually centered instead of being based on the premise of herd immunity.

As a result of the high cost of the novel vaccines currently being introduced, manufacturers will need to demonstrate the improved cost offsets and cost-effectiveness of their products over traditional vaccines. Again, additional considerations such as the relevance of an agent to the specific covered population, compliance cost offsets, quality-of-life considerations, and both patient and provider demand will be reviewed by managed care stakeholders, so legitimate value must be evident. As many of the novel vaccines being introduced are focused on preventing disease at the individual level, state governments will need to engage in dialogue with MCOs and employers to carefully delineate where there are mandates related to public health and where vaccine coverage should remain at the individual level.

Considering the emergence of myriad novel vaccines for both previously targeted and untargeted disease states, a creative and careful strategy is necessary for MCOs to maintain patient health and plan profitability. Ultimately, a sound evaluation strategy for emerging vaccines will be based on the clinical, data-driven processes employed for other pharmacotherapeutic agents, with additional considerations made at the patient, provider, and government levels. 


\section{DISCLOSURES}

This article is based on a presentation given by the author at a symposium, "The Value of Preventive Medicine: A Look at Vaccine Management," held April 11, 2007, at the Academy of Managed Care Pharmacy's 19th Annual Meeting and Showcase in San Diego, California. The symposium was supported by an educational grant from GlaxoSmithKline. The author discloses that he has received an honorarium for participation in the presentation and in this supplement. He discloses no potential bias or conflict of interest relating to this article.

\section{REFERENCES}

1. France E. Vaccine financing: the view of managed care. Am J Prev Med. $2000 ; 19(3 s): 23-25$

2. National Vaccine Advisory Committee. Strategies to sustain success in childhood immunizations. JAMA. 1999;282:363-70.

3. Commonwealth of Massachusetts Department of Health. Immunization program. Available at: http://www.mass.gov/dph/cdc/epii/imm/imm.htm. Accessed April 12, 2007.

4. Anonymous. MedImmune presents new data from pediatric studies with FluMist at pediatric academic societies annual meeting. MedImmune Web site. Available at: http://phx.corporate-ir.net/phoenix.zhtml?c=83037\&p=irol-invest ornewsArticle\&ID=849707\&highlight. Accessed July 24, 2007.
5. Centers for Disease Control and Prevention. Vaccines and immunizations. Available at: http://www.cdc.gov/vaccines/vpd-vac/varicella/default.htm. Accessed April 12, 2007.

6. National Cancer Institute. Human papillomavirus vaccines: questions and answers. Available at: http://www.nci.nih.gov/cancertopics/factsheet/risk/HPVvaccine. Accessed July 2, 2007.

7. Gardasil [package insert] Whitehouse, NJ: Merck \& Co; 2007.

8. Gianni SL, Hanson E, Moris P, et al. Enhanced humoral and memory B cellular immunity using HPV16/18 Ll VLP vaccine formulated with the MPL/aluminum salt combination (AS04) compared to aluminum salt. Vaccine. 2006;24:5937-49.

9. U.S. National Institutes of Health. ClinicalTrials.gov. Available at: http:// www.clinicaltrials.gov/ct/search?term=hpv\&submit=Search. Accessed July 2 , 2007.

10. EDP24. Teen cancer jab hailed. Available at: http://www.time.com/nation/ article/0,8599,1206813,00.html. Accessed August 13, 2007. 